The American Board of Medical Genetics is proud to acknowledge the following individuals who achieved certification in 2009.

\author{
Clinical Biochemical Geneticists \\ Baker, Mei \\ Botto, Lorenzo \\ Brunetti-Pierri, Nicola \\ Chapman, Kimberly \\ Cusmano-Ozog, Kristina \\ EL-Gharbawy, Areeg \\ Gropman, Andrea \\ He, Miao \\ James, Philip \\ Jethva, Reena \\ Manoli, Erini \\ Martin, Madelena Michele \\ Merideth, Melissa \\ Pollard, Laura \\ Sondheimer, Neal John
}

\section{Clinical Cytogeneticists}

Bi, Weimin

Brailey, Lisa Louise

Burnside, Rachel

Conlin, Laura

Crotwell, Patricia

Dai, Zunyan

Dastane, Aditi

Deak, Kristen

Geiersbach, Katherine

Kanehira, Kazunori

Kantarci, Sibel

Kogan, Jillene

Laffin, Jennifer

Lennon, Patrick

Li, Fen Catherine

Li, Feng

Liu, Jinglan

Liu, Kenian

Mehta, Indira

Michalowski, Susan

Mito, Yoshiko

Mnayer, Laila

Moore, Stephen

Nandula, Venkata

Nooraie, Farzad

Ouahchi, Karim

Pardo-Reoyo, Sherly

Rossi, Michael

Santani, Avni

Scott, Stuart

Shao, Lina

Singh, Sunita

Subramaniyam, Shivakumar

Toruner, Gokce

Wang, Jia-Chi

Xiang, Bixia

Yatsenko, Svetlana

\section{Clinical Geneticists}

AhMew, Nicholas

Alfardan, Jafar

Aubertin, Gudrun

Bernstein, Jonathan

Borowski, Kristi

Brautbar, Ariel

Burrow, Thomas
Carrillo-Carrasco, Nuria

Chapman, Kimberly

Cohen-Pfeffer, Jessica

Dagli, Aditi

Dhar, Shweta

Dugan, Sarah

Erez, Ayelet

Fickie, Matthew

Franco, Luis

Gavrilova, Ralitza

Goodin, Kara

Griffith, Christopher

Haldeman-Englert, Chad

Hauser, Natalie

Heese, Bryce

Hegde, Vijay

Herman, Kristin

Hickey, Scott

Hoffman, Benjamin

Holt, Jonathan

Hulick, Peter

Hung, Irene

Jethva, Reena

Josefsberg Ben-Yehoshua, Sagi

Kaplan, Julie

Karimov, Catherine

Kirmani, Salman

Kirmse, Brian

Konczal, Laura

Kozel, Beth

Laukaitis, Christina

Lee, Teresa

Longman, Ryan

Manace, Leslie

Manickam, Kandamurugu

Manoli, Eirini

McGregor, Tracy

McGuire, Peter

Murrain, Jr., Luis

Narumanchi, TaraChandra

Niyazov, Dmitriy

O’Brien, Barbara

Palmquist, Maria

Pena, Loren

Pickler, Laura

Powell-Hamilton, Nina

Rajadhyaksha, Aparna

Rieley, Margaret

Rink, Britton

Roe, AnneMarie

Saenz, Margarita

Safina, Nicole

Sathienkijkanchai, Achara

Schmid, Carrie

Schwab, Maria Divina Gracia

Shaffer, Brian

Shchelochkov, Oleg

Silvanskaya, Yelena

Sreenath Nagamani, Sandesh

Stolerman, Elliot

Turner, Clesson

Turocy, John

Vora, Neeta

Warnock, Ashley
Yusupov, Roman

Zarate, Yuri

Clinical Molecular Geneticists

Bai, Shaochun

Basran, Raveen

Bender, Ryan

Best, Daniel Hunter

Chen, Margaret

Chiang, Pei-Wen

Collins, Christin

Deignan, Joshua

Fernandes, Pricilla Helen

Ghazalpour, Anatole

Haverfield, Eden

Hung, Irene

Joseph, Sumy

Kimani, Jane

Kyriakopoulou, Garyfallia

Lee, Jennifer

Liu, Cong

Liu, Liu

Mancini, Deborah

Mar-Heyming, Rebecca

Mehta, Indira

Morra, Massimo

Oshima, Junko

Pyatt, Robert

Reshmi, Shalini

Riley-Gillis, Bridget

Rossi, Michael

Sathienkijkanchai, Achara

Sperber, Steven

Sun, Guoli

Svensson, Annika

ten Bosch, John

Toruner, Gokce

Turner, Clesson

Wang, Hong

Wang, Jia-Chi

Wang, Jing

Willis, Alecia

Yatsenko, Alexander

Zou, Ying

Zvereff, Val

Subspecialty: Medical Biochemical Geneticists (This certification was offered for the first time in 2009)

Champaigne, Neena

Descartes, Maria

Feigenbaum, Annette

Freedenberg, Debra

Galvin-Parton, Patricia

Goodin, Kara

Graham, Brett

Grange, Dorothy

Gucsavas-Calikoglu, Muge

Jiang, Yong-hui

Madan-Khetarpal, Suneeta

Palmer, Susan

Shinawi, Marwan

Yang, Samuel 